\title{
Bioequivalence of two quetiapine extended release tablets in Chinese healthy volunteers under fasting and fed conditions and effects of food on pharmacokinetic profiles
}

This article was published in the following Dove Press journal:

Drug Design, Development and Therapy

\author{
Xiaomei Huang' \\ Suhua Zhang ${ }^{2,3}$ \\ Yanxia Ma' \\ Heng Yang' \\ Chuan $\mathrm{He}^{\prime}$ \\ Rufang Tian' \\ Han Mei' \\ Lipeng Liu' \\ Bikui Zhang ${ }^{1-3}$ \\ 'Department of National Drug \\ Clinical Trial Research Center, \\ Xiangya Boai Rehabilitation Hospital, \\ Changsha, People's Republic of \\ China; ${ }^{2}$ Department of Pharmacy, \\ The Second Xiangya Hospital of \\ Central South University, Changsha, \\ People's Republic of China; ${ }^{3}$ Institute \\ of Clinical Pharmacy, Central South \\ University, Changsha, People's \\ Republic of China
}

Correspondence: Bikui Zhang; Lipeng Liu Department of National Drug Clinical Trial Research Center, Xiangya Boai Rehabilitation Hospital, No 61 Wanjiali North Road, Changsha 4I0I00, People's Republic of China

$\mathrm{Tel}+86$ I39 73 I 687I;

+86 I82 73।| 7465

Email 505995@csu.edu.cn;

IIp686@I26.com
Objective: The objectives of this study were to evaluate the bioequivalence of Quesero extended release (Quesero XR) tablets and Seroquel extended release (Seroquel XR) tablets under fasting and fed conditions and to determine the effect of food on the pharmacokinetic (PK) properties of Quesero XR or Seroquel XR in Chinese healthy volunteers.

Methods: A single-site, randomized, open-label, two-period crossover design with a 10-day washout period was conducted in 20 subjects under the fed and fasting studies. A single oral dose of $200 \mathrm{mg}$ Quesero XR or Seroquel XR was given to the subjects after an overnight fast of 10 hours. Blood samples were taken at scheduled time spots from 0 hour pre dose to 36 hours post dose. Plasma concentrations of quetiapine were measured by a validated ultraperformance liquid chromatography-tandem mass spectrometry (UPLC-MS/MS) method. The PK parameters were calculated by non-compartment analysis using Phoenix WinNonlin software.

Results: On both conditions, no significant differences were found among the main PK parameters of the two preparations by analysis of variance $(P>0.05)$; the Wilcoxon test of maximum peak plasma concentration $\left(\mathrm{T}_{\max }\right)$ showed no significant differences $(P>0.05)$; the $90 \%$ confidence limit (CL) of $\operatorname{lnC}_{\max }, \operatorname{lnAUC}_{0 \rightarrow 36}$, and $\ln \mathrm{AUC}_{0 \rightarrow \infty}$ fell within the acceptable range of $80 \%-125 \%$. As compared with the fasting state, the $\mathrm{T}_{\max }$ was advanced and the mean maximum plasma concentration $\left(\mathrm{C}_{\max }\right), \mathrm{AUC}_{0 \rightarrow 36}$, and $\mathrm{AUC}_{0 \rightarrow \infty}$ were also increased in the fed state; the geometric mean ratio and $90 \% \mathrm{CI}$ of the main $\mathrm{PK}$ parameters fell outside the range of the CIs; analysis of variance showed significant differences in the other PK parameters except for apparent total clearance after oral administration (clearance rate; $P<0.05$ ).

Conclusion: The two formulations of Quesero XR and Seroquel XR are bioequivalent under both fasting and fed conditions, and food may affect the PK profiles by increasing the rate and extent of absorption of Quesero XR or Seroquel XR in Chinese healthy volunteers.

Keywords: quetiapine, UPLC-MS/MS, food effect, pharmacokinetics

\section{Introduction}

Quetiapine (Figure 1), a novel benzodiazepine derivative, is an atypical psychotropic drug. ${ }^{1}$ Quetiapine was developed by AstraZeneca Pharmaceuticals in 1985 and was approved by the US Food and Drug Administration (FDA) in 1997. ${ }^{2,3}$ Currently, it has been listed in more than 80 countries around the world. The formulations of quetiapine, both immediate release (IR) and extended release (XR) tablets, ${ }^{4}$ are widely used in clinical treatment of schizophrenia, ${ }^{3,5-7}$ bipolar disorder, ${ }^{8-10}$ depressive disorder, ${ }^{11-13}$ 


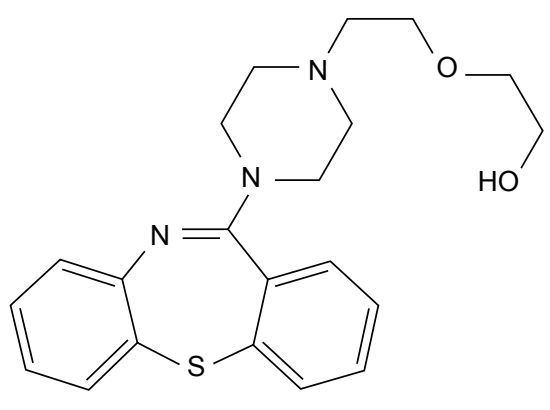

Figure I Chemical structural formula of quetiapine.

and conduct disorder. ${ }^{14}$ Quetiapine has the antipsychotic, antidepressant, anxiolytic, and sedative pharmacological effects and also reduces the incidence of extrapyramidal symptoms. ${ }^{1,15,16}$

Quetiapine XR, first approved by the FDA and named as Seroquel XR, as compared with other antipsychotics, brings better patient compliance, smaller oral dosage once daily, fewer side effects, better safety, tolerance, and effectiveness. ${ }^{1,16}$ So, there has been a growing interest in the clinical application of quetiapine XR. Meanwhile, many generic drugs of quetiapine XR have emerged. For instance, Quesero XR tablet $200 \mathrm{mg}$ was registered in Hong Kong by Europharm Laboratories Co., Ltd. in 2017, which was a generic drug of quetiapine XR. As we all know, before the generic drugs are licensed in the medical markets, bioequivalence (BE) study remains important. Few literatures have been found on the BE study of quetiapine XR in Chinese healthy subjects.

Quetiapine was classified as a Biopharmaceutics Classification System (BCS) class II drug, with a low solubility and high permeability, and was absorbed well and quickly after oral administration in humans. Some papers reported the time to the maximum peak plasma concentration $\left(\mathrm{T}_{\max }\right)$ of quetiapine IR to be $0.6-1.5$ hours, ${ }^{1,17,18}$ while that of quetiapine XR to be 5-6 hours. ${ }^{1,19,20}$ The elimination half-life $\left(t_{1 / 2}\right)$ of quetiapine was 7-12 hours. ${ }^{17-20}$ According to the study of Bakken et al, ${ }^{21}$ quetiapine is primarily metabolized by CYP450 3A4 in the liver, and the main metabolites include quetiapine sulfoxide, $N$-desalkylquetiapine, 7-hydroxyquetiapine, and $O$-desalkylquetiapine. However, all other metabolites are inactive except for $\mathrm{N}$-desalkylquetiapine. ${ }^{22}$ Quetiapine is mostly excreted in the urine. ${ }^{22}$ The plasma protein binding rate of quetiapine is $83 \%$, with an apparent volume of distribution $\left(V_{\mathrm{d}}\right)$ of $10 \mathrm{~L} / \mathrm{kg} .{ }^{23}$ As reported, the effect of food on the pharmacokinetics (PKs) of quetiapine XR lies on whether the food is of high fat or low fat, ${ }^{20}$ indicating that high-fat food increased the mean maximum plasma concentrations $\left(\mathrm{C}_{\max }\right)$ at steady state $\left(\mathrm{C}_{\max , \mathrm{ss}}\right)$ by about $20 \%$ at a daily dose of 200 and $300 \mathrm{mg}$ quetiapine XR in patients. ${ }^{20}$ The effect of food suggests an increase in the bioavailability of both the $50 \mathrm{mg}$ and the $300 \mathrm{mg}$ quetiapine XR after a high-fat meal, and the $\mathrm{AUC}_{0 \rightarrow 24}$ was increased for both strengths by around $20 \%$ after high-fat meal in patients. ${ }^{20,24}$ However, low fat did not affect the PKs of $50 \mathrm{mg}$ quetiapine XR in healthy humans and $300 \mathrm{mg}$ quetiapine XR in the patients..$^{20,24}$ No report has been found on the effect of food on the PK properties of quetiapine XR in Chinese healthy volunteers.

This paper mainly aims to evaluate the BE of Quesero XR (the trial preparation [T]) and Seroquel XR (the reference preparation $[R]$ ) in Chinese healthy volunteers under fasting and fed conditions with a validation quantitative analysis of quetiapine by ultra-performance liquid chromatographytandem mass spectrometry (UPLC-MS/MS). We also assessed the effect of food on the PK properties of Quesero XR or Seroquel XR in Chinese healthy volunteers.

\section{Subjects and methods Subject selection}

The study was designed in accordance with the Declaration of Helsinki, Good Clinical Practice (GCP) guidelines, and other related guiding principles. The study protocol was reviewed and approved by the independent ethics committee of Xiangya Boai Rehabilitation Hospital. The subjects were selected according to the inclusion and exclusion criteria.

The inclusion criteria were as follows: both males and females aged $>18$ years; body weight more than $55 \mathrm{~kg}$ and $45 \mathrm{~kg}$ for males and females, respectively; and body mass index at $19-26 \mathrm{~kg} / \mathrm{m}^{2}$, including the boundary value. All the subjects signed the informed consent.

The exclusion criteria covered history of or suffering from any serious diseases at present; allergy; surgical operations 4 weeks before the study or planned surgery during the study; medication taking 14 days before the study; blood donation and participation in any clinical trials 3 months before the study; excess coffee, tea, caffeinated beverage, or alcohol drinkers per day; smokers with more than five cigarettes per day; abnormal alcohol breathing test; drug abusers and positive drug abuse screening test (morphine, methamphetamine, ketamine, ecstasy, and cannabis); abnormal vital signs (systolic pressure $<90 \mathrm{mmHg}$ or $>140$ $\mathrm{mmHg}$; diastolic pressure $<50 \mathrm{mmHg}$ or $>90 \mathrm{mmHg}$; heart rate $<50 \mathrm{bpm}$ or $>100 \mathrm{bpm}$ ); physical examination, electrocardiogram, or laboratory examination results of clinical significance (according to the physician's judgment); unable to take valid contraception; may not complete this study for some other reasons; or those the researchers think should be excluded. 
All subjects were fully advised of the nature, purpose, procedures, and possible risks of the study by the investigators. All subjects signed the informed consent before the study.

\section{Drugs, chemicals, and reagents}

T, Quesero XR tablet $200 \mathrm{mg}$ (containing $200 \mathrm{mg}$ quetiapine per tablet, lot: G075170726, expiry: June 2019, Hong Kong registration no.: HK-65348), was manufactured by Europharm Laboratories Co., Ltd. (Tai Po, Hong Kong); R, Seroquel XR tablets $200 \mathrm{mg}$ (containing $200 \mathrm{mg}$ quetiapine per tablet, lot: 60024255, expiry: June 2019, Hong Kong registration no.: HK-57716), was manufactured by AstraZeneca Pharmaceuticals LP (USA). Both T and R were provided by the sponsor.

The materials used were quetiapine fumarate standard (United States Pharmacopoeia, lot: R065W0, content: 99.9\%), quetiapine-D8 fumarate standard (internal standard [IS], lot: 1118-048A1, content: 98.9\%), methanol and acetonitrile (HPLC grade; EMD Millipore, Billerica, MA, USA), formic acid (HPLC grade; ACS, Wilmington, DE, USA); ammonium acetate (analytical reagent grade; Sinopharm Shanghai, China), purified water by the water purification system (Milli-Q Direct 8; EMD Millipore), etc.

\section{Study design}

This study was performed with a single-center, randomized, open-label, two-period crossover trial design, including both fasting and fed BE studies. A total of 40 eligible healthy subjects were enrolled in the study, 20 in the fasting study and 20 in the fed study.

\section{Fasting BE study}

All the 20 subjects were hospitalized in site 1 day before the trial. Examination was conducted in line with the study protocol before randomizing into two groups (group 1 and group 2). The subjects were asked to fast for at least 10 hours before the oral administration. All of them underwent two study periods: taking a single oral dose of $200 \mathrm{mg}$ quetiapine XR (one trial preparation or one reference preparation) with $240 \mathrm{~mL}$ water. Group 1 was orally administered one trial preparation, while group 2 was given one reference preparation. The two study periods were separated by a washout period of 10 days before they took the other preparation in turn.

After the administration, the hands and mouths of all subjects were checked. All subjects were required to keep their body upright and accompanied by the investigator to the toilet 4 hours after the administration. Drinking water was not allowed from 1 hour pre dose to 2 hours post dose except when needed for drug administration. Standardized meals were provided to all subjects 4 and 10 hours after the administration. The same food was served to all the subjects during the trial, and the recipe was consistent in the two periods of the study.

\section{Fed BE study}

The other 20 eligible subjects participated in the fed BE study. All the procedures were the same as those in the fasting group, except for fasting for at least 10 hours before taking high-fat and high-calorie meals at about $800-1,000 \mathrm{kcal}$ (approximately $150 \mathrm{kcal}$ protein, $250 \mathrm{kcal}$ carbohydrates, and 500-600 kcal fat). The subjects finished their meals within 30 minutes (eating all food) before taking drugs. Other processes were similar to those of the fasting state.

\section{Sample collection}

Under both fasting and fed conditions, before taking the drug (0 hour) and at $0.5,1,1.5,2,3,4,5,6,7,8,9,10,12$, 16,24 , and 36 hours post dose, $4 \mathrm{~mL}$ of the venous blood was taken. Ten days later, the two groups under each condition were given reverse preparation and the same amount of blood $(4 \mathrm{~mL})$ was taken at the same time points. The blood samples were centrifuged in a vacuum blood tube containing sodium heparin to separate the plasma and stored at $-60^{\circ} \mathrm{C}$ to $-90^{\circ} \mathrm{C}$ until testing.

\section{Analytical methods}

Concentration of quetiapine was quantitatively analyzed by a validated method of UPLC system (I-Class; Waters Corporation, Milford, MA, USA) equipped with an Xevo TQD tandem triple quadrupole mass spectrometer (Waters). Chromatographic separation was performed on an analytical column (Waters ACQUITY UPLC ${ }^{\circledR}$ BEH Phenyl $1.7 \mu \mathrm{m}$, $2.1 \times 50 \mathrm{~mm}$ ) with an in-line filter holder (Waters CRITICAL CLEAN $\left.^{\mathrm{TM}}\right)$. The analytical mobile phase included the water phase (containing $10 \mathrm{mM}$ ammonium acetate and $0.3 \%$ formic acid) and acetonitrile (v/v, 70:30) and was performed in an isocratic elution mode. The flow rate of the analytical pump was $0.5 \mathrm{~mL} \cdot \mathrm{min}^{-1}$, the column temperature was maintained at $40^{\circ} \mathrm{C}$, and the temperature of autosampler was set at $15^{\circ} \mathrm{C}$.

Mass spectrometer was manipulated by a ZSpray ${ }^{\mathrm{TM}}$ electrospray ionization (ESI) equipped with a positive ion polarity. Biologic sample quantification was accomplished in multiplex reaction monitoring (MRM) or selected ion monitoring mode. The optimized operating parameters were as follows: ion source temperature, $150^{\circ} \mathrm{C}$; 
capillary voltage, $0.5 \mathrm{kV}$; desolvent gas flow, 1,000 L/h; and desolvent temperature, $500^{\circ} \mathrm{C}$; the precursor-product ion transitions monitored were $\mathrm{m} / \mathrm{z} 384.10 \rightarrow 253.14$ for quetiapine and $\mathrm{m} / \mathrm{z} 392.15 \rightarrow 258.19$ for quetiapine-D8 (the IS). The cone potential for quetiapine was $44 \mathrm{~V}$ and that for quetiapine-D8 was $50 \mathrm{~V}$. The collision energy for quetiapine was $20 \mathrm{eV}$ and that for quetiapine-D8 was $24 \mathrm{eV}$. All the data were collected through Unifi Data Process Software (Waters).

\section{Sample preparation}

The plasma samples were processed by adding $600 \mu \mathrm{L}$ IS precipitant (prepared with $50 \%$ methanol) into $200 \mu \mathrm{L}$ of the plasma sample, vortexing for 30 seconds, and centrifugating at $18,400 \times g$ for 6 minutes before $2 \mu \mathrm{L}$ of the supernatant was injected into the UPLC-MS/MS system for analysis.

\section{Method validation}

We established and validated a method to determine quetiapine by UPLC-MS/MS, in the light of FDA and China Food and Drug Administration-related guidelines. ${ }^{25,26}$ The method of validation included specificity, linearity, lower limit of quantification (LLOQ), matrix effect, extraction recovery, accuracy, precision, stability (storage stability of the autosampler, bench-top stability, freeze-thaw stability, and long-term storage stability), and the quality control (QC) during the subject sample analysis.

\section{Safety assessment}

Safety of the preparation was evaluated by closely monitoring the vital signs (blood pressure, pulse, and temperature) of the subjects at screening, baseline, 0 hour pre dose, and 2 , $4,8,12$, and 24 hours after the oral administration. Physical examination, laboratory examination, and electrocardiogram were conducted at the screening and at the end of study. Adverse events during the trial were recorded in the source document. Experienced doctors, nurses, and researchers trained by GCP observed the entire study.

\section{PK parameters and statistical analyses}

The mean plasma concentration-time curves for each subject at each sampling time under each condition were plotted. $\mathrm{T}_{\max }$ and $\mathrm{C}_{\max }$ were obtained from the concentration-time curves. $K_{\mathrm{e}}$ was the slope of a linear regression from the elimination time vs concentration curve after the logarithmic conversion. $\mathrm{t}_{1 / 2}$ was calculated as $0.693 / K_{\mathrm{e}}$. The time of the last measurable concentration was $\mathrm{t}_{\mathrm{n}}$. $\mathrm{AUC}_{0 \rightarrow \mathrm{t}_{\mathrm{n}}}$ (area under the plasma concentration-time curve from time 0 to $t_{n} h$ ) was calculated following the trapezoidal rule. $\mathrm{AUC}_{0 \rightarrow \infty}$ (area under the plasma concentration-time curve from time 0 to infinite time) was calculated as $\mathrm{AUC}_{0 \rightarrow \mathrm{t}_{\mathrm{n}}}+C_{\mathrm{t}_{\mathrm{n}}} / \mathrm{K}_{\mathrm{e}} \cdot \mathrm{MRT}_{0 \rightarrow \infty}$ (residence time) was calculated as $\mathrm{AUMC}_{0 \rightarrow \infty} / \mathrm{AUC}_{0 \rightarrow \infty} . \mathrm{Vz} / \mathrm{F}$ (the apparent volume of distribution) and apparent total clearance after oral administration $(\mathrm{CL} / \mathrm{F})$ (clearance rate) were calculated as single dose $/\left(\mathrm{K}_{\mathrm{e}} \times \mathrm{AUC}_{\mathrm{inf}}\right)$ and single dose/ $\mathrm{AUC}_{\text {inf }}$ value, respectively. The relative bioavailability was calculated as $\left(\mathrm{AUC}_{0 \rightarrow \infty}[\right.$ test $\left.]\right) /\left(\mathrm{AUC}_{0 \rightarrow \infty}[\right.$ reference $\left.]\right) \times 100 \%$.

After the logarithmic conversion, the main $\mathrm{PK}$ parameters underwent analysis of variance (ANOVA) to obtain the SD in different groups. $\mathrm{T}_{\text {max }}$ underwent nonparametric Wilcoxon test; two one-sided $t$-tests were conducted to determine the BE. If the $90 \%$ confidence limit (CL) of $\mathrm{AUC}_{0 \rightarrow \mathrm{t}_{\mathrm{n}}}, \mathrm{AUC}_{0 \rightarrow \infty}$, and $\mathrm{C}_{\max }$ for the trial and reference preparations falls within $80.00 \%-125.00 \%$, we may conclude that the two preparations are bioequivalent. The effect of food on the PK profiles of Quesero XR or Seroquel XR was determined by comparing the $P$-value of ANOVA of the fed state with that of the fasting state and the geometric mean ratio (GMR) and $90 \%$ $\mathrm{CI}$ of the fed treatment to the fasting treatment for the main PK parameters. We used scheduled PK time for calculation of PK parameters. Phoenix WinNonlin software (Version 8.0) was used in the statistical analysis.

\section{Results}

\section{Subject information}

Totally 40 subjects were qualified for the study. According to the order of signing informed consent, all eligible subjects were randomized into two teams: one for the fasting condition and the other for the fed condition. Their demographic characteristics were listed as follows. The fasting study comprised 20 healthy subjects (14 males and six females) aged 18-43 years (27.2 \pm 7.0 years), weighting 46.5-76 kg $(62.3 \pm 7.5 \mathrm{~kg})$, and height ranging $150-179 \mathrm{~cm}(164.2 \pm 8.5 \mathrm{~cm})$. The fed study comprised the other 20 healthy subjects (14 males and six females) aged 19-42 years (26.2 \pm 5.4 years), weighing $47.3-85$ $\mathrm{kg}(61.4 \pm 9.8 \mathrm{~kg})$, and height ranging 150.5-182.5 $\mathrm{cm}$ $(165.4 \pm 9.5 \mathrm{~cm})$.

Before being hospitalized, the females were asked to take blood $\beta$-human chorionic gonadotropin examination. All the subjects had physical examinations, laboratory tests, and other necessary tests. All subjects were non-drinkers and non-smokers without abnormalities detected by the physical examinations and laboratory tests. All subjects had no history of drug allergy or drug dependence, no chronic illnesses, and had not taken any medications before the trial. During the trial, all subjects were catered a bland diet without prescribing drugs other than the preparations being studied. No tobacco, 
alcohol, or drink with caffeine was allowed. In the fasting state, two subjects quit the study due to personal reasons.

\section{Method validation}

A UPLC-MS/MS method was established and validated to detect quetiapine in human blood plasma. Method specificity was tested by comparing the chromatogram of blank plasma, chromatogram of blank plasma with quetiapine and the IS, and chromatogram of plasma sample after the administration in the subjects. The retention time of quetiapine and the IS was 0.94 and 0.92 minutes, respectively. The plasma-derived substances and other impurities did not interfere the separation and determination of the sample.

Good linearity was found at $1-500 \mathrm{ng} \cdot \mathrm{mL}^{-1}$. The regression equation was $Y=0.0606 X+0.000375(r=0.99985)$. The lower limit of quantitation (LOQ) of quetiapine in the plasma was $1 \mathrm{ng} \cdot \mathrm{mL}^{-1}$. Plasma samples at $1 \cdot \mathrm{ng} \cdot \mathrm{mL}^{-1}$ (LLOQ) were prepared by adding the standard solution into the blank plasma and were repeatedly measured for five times. The intraday relative recovery (relative standard deviation) of the LLOQ was $1.8 \%$.

The average matrix effect and extraction recovery of quetiapine were $99.2 \%-105.0 \%$ and $103.4 \%-105.9 \%$, respectively, at three QC concentration levels (Table 1). The average matrix effect and extraction recovery of the IS were $102.8 \% \pm 2.3 \%$ and $103.5 \% \pm 1.7 \%$ at $10 \mathrm{ng} / \mathrm{mL}$. The degree of the influence of matrix effect and extraction recovery to analytes met the requirements of biological sample analysis. The accuracy of the method ranged $99.3 \%-101.1 \%$. Both the intraday and interday relative standard deviations were less than $2.8 \%$, which met the methodological requirements of biological sample analysis.

The stability of quetiapine is summarized in Table 1, all within $95.3 \%-102.8 \%$, indicating that quetiapine was stable in human plasma under these conditions. The relative bias $\%$ of the control plasma samples with high and medium concentration levels should fall within $-15 \%$ to $15 \%$, while the bias $\%$ of low concentration level should fall within $-20 \%$ to $20 \%$. The results of the control plasma samples of every batch showed that the testing condition was stable during the whole analysis.

\section{BE studies and effect of food on PK}

The validated UPLS-MS/MS method was used to successfully measure quetiapine in Chinese healthy subjects after oral administration of the test and reference drugs under both fasting and fed conditions.

In the BE study, the mean plasma concentration-time curves of Quesero XR (T) and Seroquel XR (R) after a single oral dose of $200 \mathrm{mg}$ under both fasting and fed conditions are

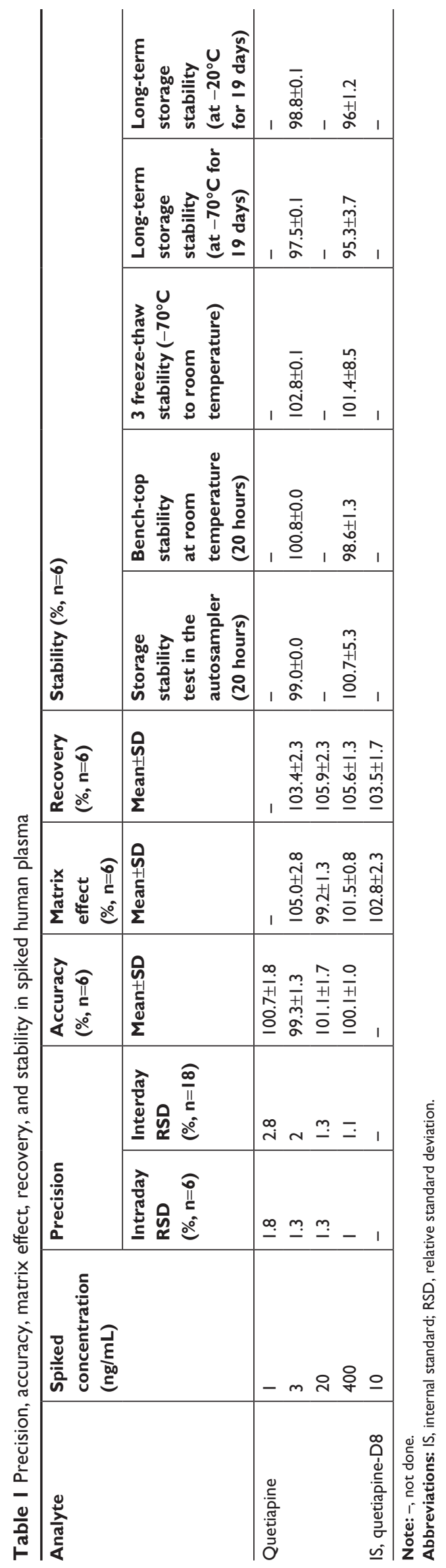




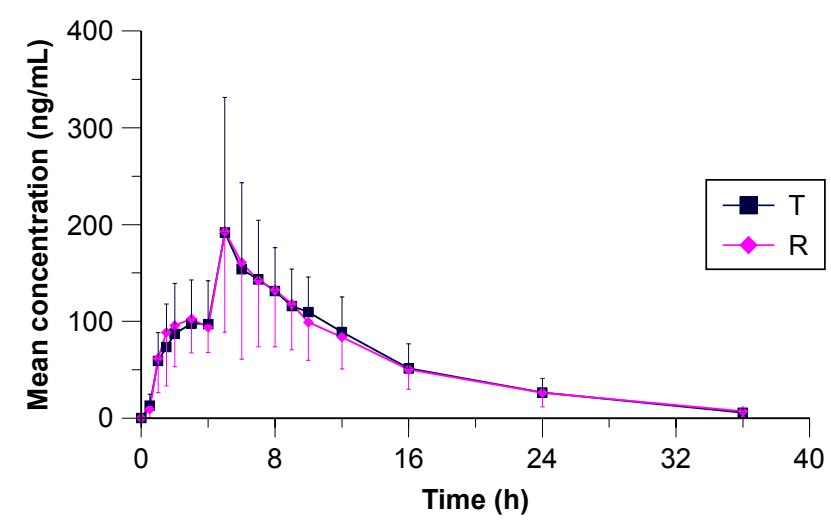

Figure 2 Mean plasma concentration-time curves of Quesero XR (T) and Seroquel $X R(R)$ after a single oral dose of $200 \mathrm{mg}$ in 18 healthy subjects under fasting conditions (mean $\pm S D, n=18$ ).

Abbreviations: Quesero XR, Quesero extended release; R, reference preparation; Seroquel $X R$, Seroquel extended release; $T$, trial preparation.

shown in Figures 2 and 3, respectively. The main PK parameters, including $\mathrm{T}_{\max }, \mathrm{C}_{\max }, \mathrm{AUC}_{0 \rightarrow 36}, \mathrm{AUC}_{0 \rightarrow \infty}$, and $\mathrm{t}_{1 / 2}$ after a single dose of $200 \mathrm{mg}$ of the Quesero XR (T) and Seroquel $\mathrm{XR}(\mathrm{R})$, are summarized in Table 2. The ANOVA of $\ln \mathrm{C}_{\max }$, $\ln \mathrm{AUC}_{0 \rightarrow 36}$, and $\ln \mathrm{AUC}_{0 \rightarrow \infty}$ and the Wilcoxon test of $\mathrm{T}_{\max }$ are also listed in Table 2, showing no significance difference between Quesero XR (T) and Seroquel XR (R) under both fasting and fed conditions. The results of the two one-sided $t$-tests and $90 \% \mathrm{CL}$ of $\operatorname{lnC}_{\max }, \operatorname{lnAUC}_{0 \rightarrow 36}$, and $\ln \mathrm{AUC}_{0 \rightarrow \infty}$ are shown in Table 3 . The $90 \%$ CL was within the acceptance range of $80 \%-125 \%$. The relative bioavailability of fasting and fed conditions was $100.2 \% \pm 2.7 \%$ and $101.2 \% \pm 2.7 \%$, respectively.

As to the effect of food on the PK, Figures 4 and 5 show the mean plasma concentration-time curves of Quesero XR (T) and Seroquel XR (R) after a single oral dose under both

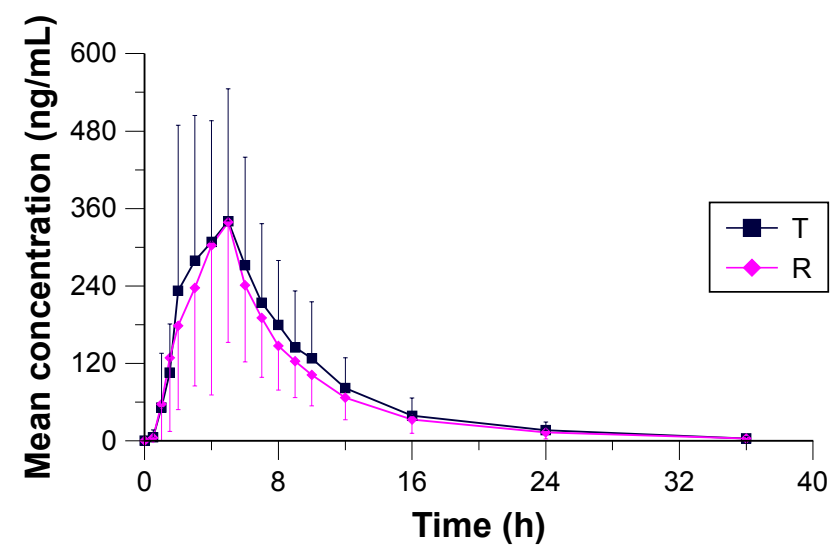

Figure 3 Mean plasma concentration-time curves of Quesero XR (T) and Seroquel $X R(R)$ after a single oral dose of $200 \mathrm{mg}$ in 20 healthy subjects under fed conditions (mean $\pm S D, n=20$ ).

Abbreviations: Quesero XR, Quesero extended release; R, reference preparation; Seroquel $X R$, Seroquel extended release; $T$, trial preparation. conditions, respectively. The main PK parameters, $P$-value of ANOVA, GMR, and $90 \% \mathrm{CI}$ of fed to fasting treatment are presented in Table 2.

\section{Safety assessment}

During the test period, many subjects showed mild side effects such as dizziness and drowsiness under both fasting and fed conditions.

For example, under fasting condition, the rates of the adverse events (AEs) occurred in the first test period were somnolence $11.1 \%(2 / 18)$, nausea $11.1 \%(2 / 18)$, dry mouth $5.6 \%(1 / 18)$, dizziness $5.6 \%(1 / 18)$, vomiting $5.6 \%(1 / 18)$, fatigue $5.6 \%(1 / 18)$, and irritability, anxiety, and chest distress $5.6 \%(1 / 18)$ - in total $55.6 \%(10 / 18)$; in the second test period, the rates of AEs were somnolence $11.1 \%(2 / 18)$, nausea $11.1 \%(2 / 18)$, dry mouth $5.6 \%(1 / 18)$, and dizziness $16.6(3 / 18)$ - in total $38.9 \%(7 / 18)$. Under the fed condition, in the first test period, the rates of AEs were somnolence $5.0 \%$ $(1 / 20)$, nausea $5.0 \%(1 / 20)$, and dizziness $10.0 \%(2 / 20)$ - in total $20 \%$ (4/20); AEs did not occur in the second period. The rates of AEs in the fed state were less than those in the fasting state, which may be because the food reduced the absorption of quetiapine. The rates in the second period were lower than those in the first period under each condition, which could be due to the subjects' tolerance to quetiapine.

The common adverse reactions ( $\geq 10 \%)$ were listed in the labeling of Seroquel $\mathrm{XR}^{\circledR}$ : somnolence, dry mouth, dizziness, etc., ${ }^{27}$ which is similar to the results of our study. All these symptoms of AEs were transient. In addition, no other severe AEs were observed during the whole study.

\section{Discussion \\ Method development}

As reported, there are many ways to quantitatively analyze quetiapine in human biological samples such as HPLC, ${ }^{28}$ HPLC-MS/MS, ${ }^{29,30}$ and liquid chromatography (LC)/MS/ MS. ${ }^{19,24,31-33}$ However, method for BE study of $200 \mathrm{mg}$ quetiapine XR in Chinese volunteers was only one; LC-MS/MS technology was used..$^{34}$ In this study, we used UPLC-MS/MS to measure quetiapine in the human plasma, considering the high-throughput sequencing, high sensitivity, strong specificity, and time-saving aspects of UPLC-MS/MS. The method separated quetiapine at 0.94 minute, which is simple, rapid, and sensitive in determining quetiapine in the human plasma.

\section{BE studies}

In all, 18 of the 20 subjects completed the study in the fasting state, while all the 20 volunteers finished the study under the 


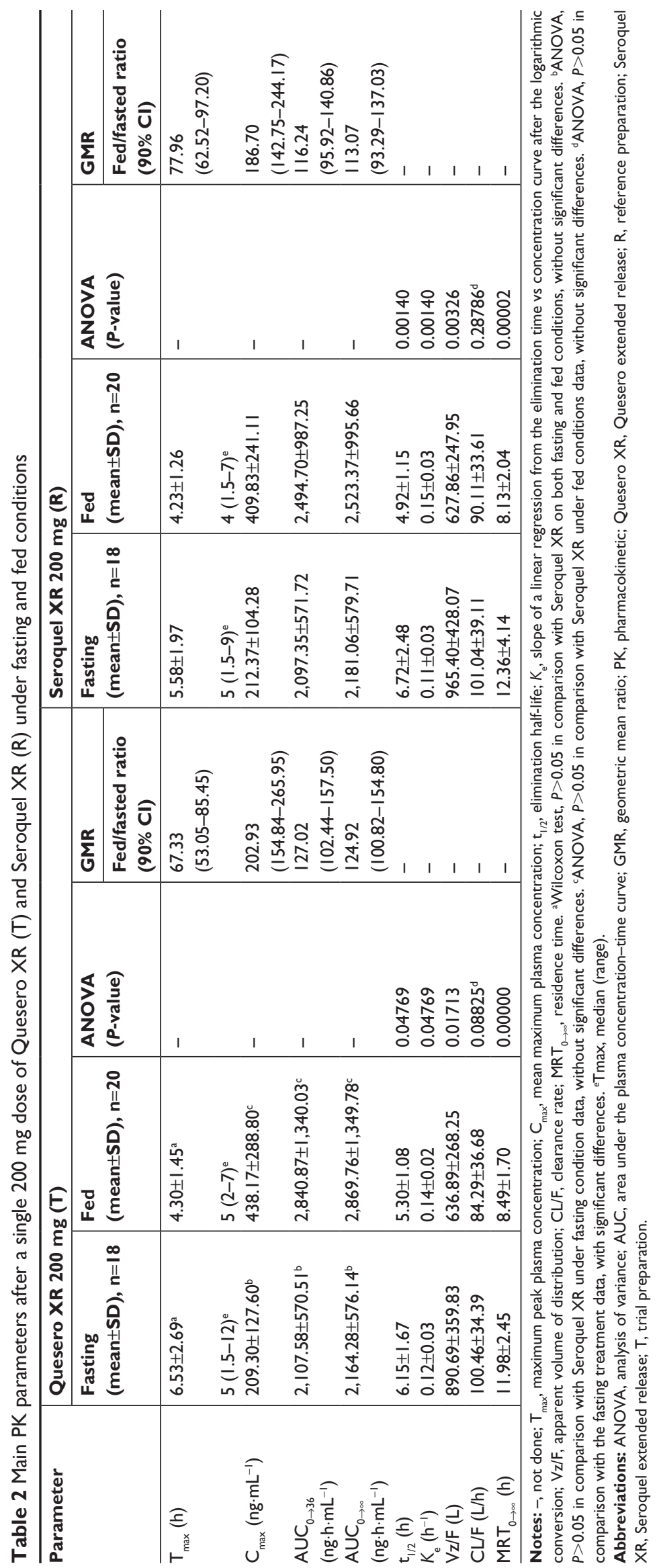


Table 3 Two one-sided $t$-tests and $90 \% \mathrm{CL}$ of $\ln C_{\max }, \ln A \cup C_{0 \rightarrow 36}$, and $\ln A U C_{0 \rightarrow \infty}$ after a single oral dose of $200 \mathrm{mg} Q$ uesero $X R(T)$ and Seroquel XR (R) under fasting and fed conditions

\begin{tabular}{|c|c|c|c|c|c|c|c|}
\hline \multirow[t]{2}{*}{ State } & \multirow[t]{2}{*}{ Parameter } & \multirow[t]{2}{*}{$\mathbf{T}_{\mathrm{L}}$} & \multirow[t]{2}{*}{$\mathbf{T}_{\mathrm{H}}$} & \multirow[t]{2}{*}{$T_{0.05}$} & \multirow[t]{2}{*}{$P$-value } & \multicolumn{2}{|l|}{$90 \% \mathrm{CL}$} \\
\hline & & & & & & 90\% CL-L & $90 \%$ CL-H \\
\hline \multirow[t]{3}{*}{ Fasting } & $\ln C_{\max }$ & 1.972 & -2.711 & $\begin{array}{l}1.746 \\
1.746\end{array}$ & $\begin{array}{l}\text { High side } P<0.05 \\
\text { Low side } P<0.05\end{array}$ & 81.74 & 114.02 \\
\hline & $\ln A \cup C_{0 \rightarrow 36}$ & 4.571 & -4.227 & $\begin{array}{l}1.746 \\
1.746\end{array}$ & $\begin{array}{l}\text { High side } P<0.05 \\
\text { Low side } P<0.05\end{array}$ & 92.33 & 110.22 \\
\hline & $\ln A \cup C_{0 \rightarrow \infty}$ & 4.711 & -4.862 & $\begin{array}{l}1.746 \\
1.746\end{array}$ & $\begin{array}{l}\text { High side } P<0.05 \\
\text { Low side } P<0.05\end{array}$ & 91.86 & 108.10 \\
\hline \multirow[t]{3}{*}{ Fed } & $\ln C_{\max }$ & 3.659 & -2.360 & $\begin{array}{l}1.734 \\
1.734\end{array}$ & $\begin{array}{l}\text { High side } P<0.05 \\
\text { High side } P<0.05\end{array}$ & 92.27 & 119.33 \\
\hline & $\ln A \cup C_{0 \rightarrow 36}$ & 6.439 & -2.524 & $\begin{array}{l}1.734 \\
1.734\end{array}$ & $\begin{array}{l}\text { High side } P<0.05 \\
\text { Low side } P<0.05\end{array}$ & 101.12 & 120.18 \\
\hline & $\ln A \cup C_{0 \rightarrow \infty}$ & 6.385 & -2.537 & $\begin{array}{l}1.734 \\
1.734\end{array}$ & $\begin{array}{l}\text { High side } P<0.05 \\
\text { High side } P<0.05\end{array}$ & 100.95 & 120.08 \\
\hline
\end{tabular}

Note: $C_{\max }$, mean maximum plasma concentration.

Abbreviations: $90 \% \mathrm{CL}, 90 \%$ confidence limit; AUC, area under the plasma concentration-time curve; Quesero XR, H, high; L, low; R, reference preparation; Quesero XR, Quesero extended release; Seroquel XR, Seroquel extended release; T, trial preparation.

fed condition. Whether in the fasting or fed state, we were able to detect the concentration of quetiapine in the human plasma 30 minutes after the oral administration, indicating rapid blood absorption of quetiapine. After the 10-day washout, we did not detect any quetiapine in the plasma, suggesting no residual effect from the first cycle of administration on the second cycle.

Whether in the fasting or fed state, the main PK parameters $\left(\mathrm{T}_{\max }, \mathrm{C}_{\max }, \mathrm{AUC}_{0 \rightarrow 36}, \mathrm{AUC}_{0 \rightarrow \infty}\right.$, and $\left.\mathrm{t}_{1 / 2}\right)$ of Quesero XR (T) and Seroquel XR (R) were similar (Table 2). No significant difference was found among the main PK parameters between the two preparations by ANOVA $(P>0.05)$. Wilcoxon test of the two formulations showed no significant difference between the $\mathrm{T}_{\max }$ values $(P>0.05)$. Although the $\operatorname{lnC}_{\max }$ ratios of several subjects fell outside $80.00 \%-125.00 \%$,

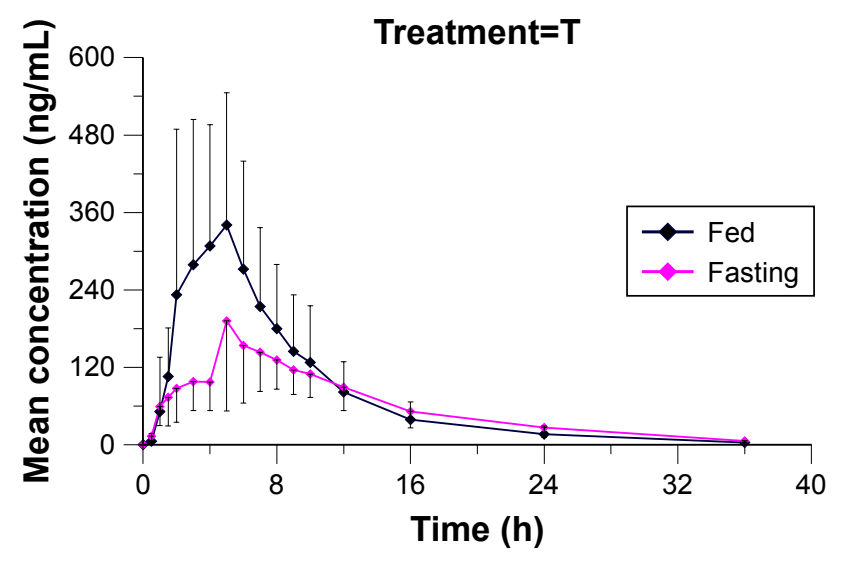

Figure 4 Mean plasma concentration-time curves of Quesero XR (T) after a single oral dose of $200 \mathrm{mg}$ in 18 healthy subjects under fasting conditions and 20 healthy subjects under fed conditions (mean $\pm S D$ ).

Abbreviations: Quesero XR, Quesero extended release; T, trial preparation. the $\mathrm{T}_{\mathrm{L}}$ and $\mathrm{T}_{\mathrm{H}}$ values were both within the critical value. The two one-sided $t$-tests also presented no significance in the main parameters, showing that with the $90 \% \mathrm{CL}$, the trial preparation $\mathrm{C}_{\text {max }}$ fell within $80.00 \%-125.00 \%$ of the reference preparation $\mathrm{C}_{\max }$. The $90 \% \mathrm{CL}$ of $\operatorname{lnC}_{\max }, \ln \mathrm{AUC}_{0 \rightarrow 36}$, and $\ln \mathrm{AUC}_{0 \rightarrow \infty}$ was within the acceptance range for BE study both in the fasting and fed states (Table 3).

In summary, we concluded that the test and reference preparations are bioequivalent under both fasting and fed conditions.

Compared with the parameters in Bui et al, ${ }^{20}$ in our study, the $\mathrm{T}_{\max }, \mathrm{C}_{\max }, \mathrm{AUC}_{0 \rightarrow 36}$, and $\mathrm{AUC}_{0 \rightarrow \infty}$ of the quetiapine under the fasting condition for the Seroquel XR (R) were lower but the $t_{1 / 2}$ was higher, while under the fed condition, in our study, the $\mathrm{t}_{1 / 2}, \mathrm{AUC}_{0 \rightarrow 36}$, and $\mathrm{AUC}_{0 \rightarrow \infty}$

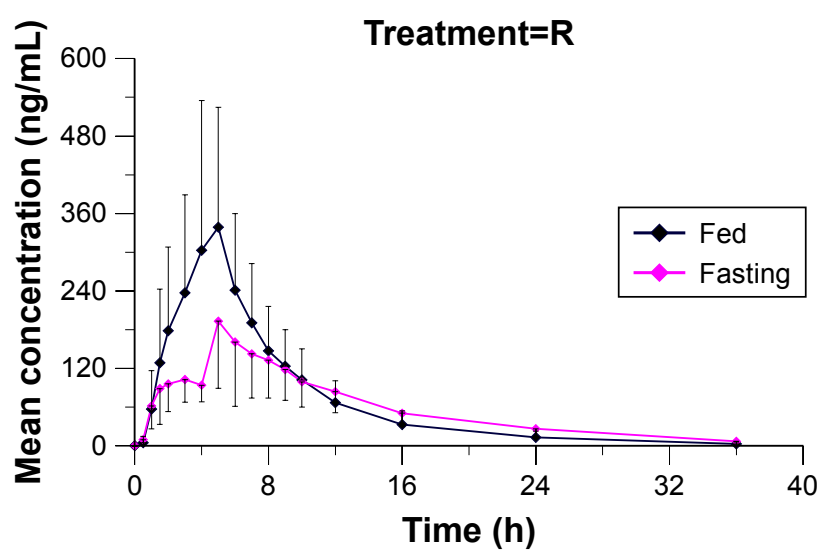

Figure 5 Mean plasma concentration-time curves of Seroquel $X R(R)$ after a single oral dose of $200 \mathrm{mg}$ in 18 healthy subjects under fasting conditions and 20 healthy subjects under fed conditions (mean $\pm \mathrm{SD}$ ).

Abbreviations: R, reference preparation; Seroquel XR, Seroquel extended release. 
were lower; the $\mathrm{C}_{\max }$ was higher; and the $\mathrm{T}_{\max }$ was similar. Compared with the study of $\mathrm{Li}$ et al, ${ }^{35}$ the $\mathrm{C}_{\max }$ and $\mathrm{AUC}_{0 \rightarrow t}$ of $300 \mathrm{mg}$ quetiapine XR in Han patients were 1.5 times to those of our study; in our study, the $\mathrm{T}_{\max }, K_{\mathrm{e}}$, and MRT were higher; however, the $t_{1 / 2}$ was a bit lower. We speculated that the different PK parameters in our study may have been caused by the different subjects, the sample size, ethnic groups, sample detector and other unknown factors, such as we studied healthy people, while Bui $\mathrm{K}$ et $\mathrm{al}^{20}$ and Li Q et $\mathrm{al}^{35}$ studied patients.

\section{Effect of food on PK profiles}

We compared the main PK parameters of Quesero XR (T) or Seroquel XR (R) in both the fasting and fed states (Table 2). For Quesero XR, $\mathrm{T}_{\text {max }}$ of the fasting and fed states was 6.5 \pm 2.7 and 4.3 \pm 1.5 hours, respectively; $\mathrm{C}_{\max }$ was $209.3 \pm 127.6$ $\mathrm{ng} \cdot \mathrm{mL}^{-1}$ and $438.2 \pm 288.8 \mathrm{ng} \cdot \mathrm{mL}^{-1}$, respectively. After the meals, $\mathrm{T}_{\max }$ was in advance and the mean $\mathrm{C}_{\max }$ almost doubled as compared with that of the fasting, demonstrating that high-calorie and high-fat meals increased the absorption rate of quetiapine. $\mathrm{AUC}_{0 \rightarrow 36}$ of the fasting and fed states was 2,107.6 \pm 570.5 and 2,840.9 $\pm 1,340.0 \mathrm{ng} \cdot \mathrm{h} \cdot \mathrm{mL}^{-1}$, respectively;

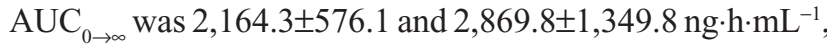
respectively. $\mathrm{AUC}_{0 \rightarrow 36}$ and $\mathrm{AUC}_{0 \rightarrow \infty}$ were all increased by $>30 \%$ after the high-fat diets, indicating that highfat diets accelerated the absorption extent of quetiapine. Similarly, for Seroquel XR in the fed or fasting condition, $\mathrm{T}_{\max }$ was advanced a bit, and $\mathrm{C}_{\max }, \mathrm{AUC}_{0 \rightarrow 36}$, and $\mathrm{AUCC}_{0 \rightarrow \infty}$ were also increased. From the original data in our study, we found that high-fat food may affect the PK profiles of quetiapine; the GMR and $90 \% \mathrm{CI}$ of food effect on $\mathrm{T}_{\max }$, $\mathrm{C}_{\max }, \mathrm{AUC}_{0 \rightarrow 36}$, and $\mathrm{AUC}_{0 \rightarrow \infty}$ in this study fell outside both equivalence intervals, which may be due to limited sample size and interindividual discrepancy in the two groups. When we compared $\mathrm{t}_{1 / 2}, K_{\mathrm{e}}, \mathrm{Vz} / \mathrm{F}, \mathrm{CL} / \mathrm{F}$, and $\mathrm{MRT}_{0 \rightarrow \infty}$ of the fed to fasting state by ANOVA, no significant difference was found in $\mathrm{CL} / \mathrm{F}(P>0.05)$, but significant differences were observed in the other parameters $(P<0.05)$.

We can see from Figures 4 and 5, under fasting condition, that the mean concentration of quetiapine was decreased a bit from 3 to 4 hours after the oral administration of the preparation. All the subjects had standard meals 4 hours post dose. After that, the mean concentration of quetiapine was rapidly increased from 4 hours post dose to 5 hours post dose; the concentration of quetiapine at 5 hours post dose was doubled to that of 4 hours post dose, which indicated that standard meals also affected the absorption of quetiapine. In contrast, former studies mentioned that low-fat food did not affect the bioavailability of quetiapine. ${ }^{20,24}$
Both high-fat food and standard meals have some effect on the PK profiles of quetiapine in Chinese healthy subjects. It is recommended to reduce food intake or have light diet at least 4 hours before taking the medicine every night once daily to avoid the influence of food on the PK profiles of Quesero XR and Seroquel XR, which is an important guidance for clinical medication safety.

\section{Conclusion}

In this paper, we successfully validated a UPLC-MS/MS method to measure quetiapine in the human plasma. The trial preparation Quesero XR is bioequivalent to the reference preparation Seroquel XR in Chinese healthy subjects under both fasting and fed conditions. We also verified that food may affect the PK profiles of Quesero XR and Seroquel XR in Chinese healthy subjects. In our study, all the subjects showed good tolerance of both preparations without any serious or unexpected adverse reactions.

\section{Acknowledgments}

We would like to heartily thank all volunteers for their contribution to the clinical trial. This study was carried out by Europharm Laboratories Co., Ltd., Hong Kong. Our sincere thanks to Zhi Tang (Changsha Duxact Biotechnology Co., Ltd., Changsha, People's Republic of China) for analyzing the samples, Wu Liang (Changsha Ruiyi Medical Technology Co., Ltd., Changsha, People's Republic of China) for data analysis, and Binbin He and Xun Tan (Changsha Ruiyi Medical Technology Co., Ltd., Changsha, People's Republic of China) for the recruitment of study subjects.

\section{Author contributions}

All authors contributed to data analysis, drafting and revising the article, gave final approval of the version to be published, and agree to be accountable for all aspects of the work.

\section{Disclosure}

The authors report no conflicts of interest in this work.

\section{References}

1. Zhang HY. Advance in the research of quetiapine fumarate extendedrelease tablets, a new formulation for atypical antipsychotics. Chin $J$ New Drugs. 2015;24(1):35-38.

2. Quetiapine fumarate. Available from: https://www.drugs.com/monograph/quetiapine-fumarate.html. Accessed June 23, 2018.

3. Riedel M, Müller N, Strassnig M, Spellmann I, Severus E, Möller HJ Quetiapine in the treatment of schizophrenia and related disorders. Neuropsychiatr Dis Treat. 2007;3(2):219-235.

4. Yaozh.com [homepage on the Internet]. [Searching "Quetiapine fumarate" in import drug database.] Available from: https://db.yaozh.com/jink ou?comprehensivesearchcontent=quetiapine\&. Accessed June 23, 2018. Chinese. 
5. Gunasekara NS, Spencer CM. Quetiapine: A Review of its Use in Schizophrenia. CNS Drugs. 1998;9(4):325-340.

6. Hui KO, Sulaiman AH, Gill JS, Pillai SK, Jambunathan ST, Rashid RA. A 3-week, open label study to evaluate the efficacy and safety of extended release quetiapine fumarate in the treatment of agitation in patients with schizophrenia. Klinik Psikofarmakoloji Bülteni-Bulletin of Clinical Psychopharmacology. 2013;23(1):14-23.

7. Naber D, Peuskens J, Schwarzmann N, et al. Subjective well-being in schizophrenia: a randomised controlled open-label 12-month non-inferiority study comparing quetiapine XR with risperidone (RECOVER). Eur Neuropsychopharmacol. 2013;23(10):1257-1269.

8. Cristancho MA, Thase ME. The role of quetiapine extended release in the treatment of bipolar depression. Adv Ther. 2010;27(11): 774-784.

9. Ketter TA, Miller S, Dell'Osso B, Wang PW. Treatment of bipolar disorder: Review of evidence regarding quetiapine and lithium. $J$ Affect Disord. 2016;191:256-273.

10. Garriga M, Solé E, González-Pinto A, et al. Efficacy of quetiapine $\mathrm{XR}$ vs placebo as concomitant treatment to mood stabilizers in the control of subthreshold symptoms of bipolar disorder: Results from a pilot, randomized controlled trial. Eur Neuropsychopharmacol. 2017; 27(10):959-969.

11. Pae CU, Sohi MS, Seo HJ, et al. Quetiapine XR: current status for the treatment of major depressive disorder. Prog Neuropsychopharmacol Biol Psychiatry. 2010;34(7):1165-1173.

12. Ignácio ZM, Calixto AV, da Silva RH, Quevedo J, Réus GZ. The use of quetiapine in the treatment of major depressive disorder: Evidence from clinical and experimental studies. Neurosci Biobehav Rev. 2018; 86:36-50.

13. Liebowitz M, Lam RW, Lepola U, Datto C, Sweitzer D, Eriksson H. Efficacy and tolerability of extended release quetiapine fumarate monotherapy as maintenance treatment of major depressive disorder: a randomized, placebo-controlled trial. Depress Anxiety. 2010;27(10): 964-976.

14. Findling RL, Reed MD, O'Riordan MA, Demeter CA, Stansbrey RJ, Mcnamara NK. Effectiveness, safety, and pharmacokinetics of quetiapine in aggressive children with conduct disorder. $J$ Am Acad Child Adolesc Psychiatry. 2006;45(7):792-800.

15. Cabaleiro T, López-Rodríguez R, Román M, et al. Pharmacogenetics of quetiapine in healthy volunteers: association with pharmacokinetics, pharmacodynamics, and adverse effects. Int Clin Psychopharmacol. 2015;30(2):82-88.

16. Peuskens J. The management of schizophrenia: focus on extended-release quetiapine fumarate. Neuropsychiatr Dis Treat. 2011;7:549-564.

17. Mahatthanatrakul W, Rattana K, Sriwiriyajan S, Wongnawa M, Ridtitid W. Bioequivalence study of a generic quetiapine in healthy male volunteers. Int J Clin Pharmacol Ther. 2008;46(9):489-496.

18. Junior EA, Duarte LF, Suenaga EM, de Carvalho Cruz A, Nakaie CR. Comparative bioavailability of two quetiapine formulations in healthy volunteers after a single dose administration. J Bioequiv Availab. 2011; 03(08): 178-181.

19. Estevez-Carrizo FE, Parrillo S, Ercoli MC, Estevez-Parrillo FT. Single-dose relative bioavailability of a new quetiapine fumarate extended-release formulation: a postprandial, randomized, open-label, two-period crossover study in healthy Uruguayan volunteers. Clin Ther. 2011;33(6):738-745.
20. Bui K, Earley W, Nyberg S. Pharmacokinetic profile of the extendedrelease formulation of quetiapine fumarate (quetiapine XR): clinical implications. Curr Med Res Opin. 2013;29(7):813-825.

21. Bakken GV, Rudberg I, Christensen H, Molden E, Refsum H, Hermann M. Metabolism of quetiapine by CYP3A4 and CYP3A5 in presence or absence of cytochrome B5. Drug Metab Dispos. 2009; 37(2):254-258.

22. Devane CL, Nemeroff CB. Clinical pharmacokinetics of quetiapine: an atypical antipsychotic. Clin Pharmacokinet. 2001;40(7):509-522.

23. Flammia DD, Valouch T, Venuti S. Tissue distribution of quetiapine in 20 cases in Virginia. J Anal Toxicol. 2006;30(4):287-292.

24. FDA. Clinical pharmacology and biopharmaceutics reviews for Seroquel XR; 2006. Available from: https://www.accessdata.fda. gov/drugsatfda_docs/nda/2007/022047Orig1s000ClinPharmR.pdf. Accessed June 23, 2018.

25. FDA [homepage on the Internet]. Analytical procedures and methods validation for drugs and biologics-guidance for industry; 2015. Available from: https://www.fda.gov/downloads/drugs/guidancecomplianceregulatoryinformation/guidances/ucm386366.pdf. Accessed June 23, 2018.

26. CFDA [homepage on the Internet]. Technical guidelines for the study of human bioavailability and bioequivalence of chemical drug preparations; 2005. Available from: http://www.cde.org.cn/zdyz. do?method=largePage\&id=2070. Accessed June 23, 2018.

27. FDA [homepage on the Internet]. Highlights of prescribing information; 2017. Available from: $\mathrm{https}: / / \mathrm{www}$. accessdata.fda.gov/drugsatfda_docs/ label/2017/022047s039lbl.pdf. Accessed June 24, 2018.

28. Mandrioli R, Fanali S, Ferranti A, Raggi MA. HPLC analysis of the novel antipsychotic drug quetiapine in human plasma. JPharm Biomed Anal. 2002;30(4):969-977.

29. Barrett B, Holcapek M, Huclová J, et al. Validated HPLC-MS/MS method for determination of quetiapine in human plasma. J Pharm Biomed Anal. 2007;44(2):498-505.

30. Park S, Park CS, Lee SJ, et al. Development and validation of a high-performance liquid chromatography-tandem mass spectrometric method for simultaneous determination of bupropion, quetiapine and escitalopram in human plasma. Biomed Chromatogr. 2015;29(4): 612-618.

31. Pan RN, Kuo BP, Pao LH. Validated LC-MS-MS method for the determination of quetiapine in human plasma: application to a pharmacokinetic study. J Chromatogr Sci. 2012;50(3):277-282.

32. Xiong X, Yang L, Duan J. Development and validation of a sensitive and robust LC-MS/MS with electrospray ionization method for simultaneous quantitation of quetiapine and its active metabolite norquetiapine in human plasma. Clin Chim Acta. 2013;423:69-74.

33. Davis PC, Bravo O, Gehrke M, Azumaya CT. Development and validation of an LC-MS/MS method for the determination of quetiapine and four related metabolites in human plasma. J Pharm Biomed Anal. 2010;51(5):1113-1119.

34. Li M, Zhang S, Shi A, Qi W, Liu Y. Determination of quetiapine in human plasma by LC-MS/MS and its application in a bioequivalence study. J Chromatogr B Analyt Technol Biomed Life Sci. 2017;1060: $10-14$.

35. Li Q, Su YA, Liu Y, et al. Pharmacokinetics and tolerability of extendedrelease quetiapine fumarate in Han Chinese patients with schizophrenia. Clin Pharmacokinet. 2014;53(5):455-465.
Drug Design, Development and Therapy

\section{Publish your work in this journal}

Drug Design, Development and Therapy is an international, peerreviewed open-access journal that spans the spectrum of drug design and development through to clinical applications. Clinical outcomes, patient safety, and programs for the development and effective, safe, and sustained use of medicines are the features of the journal, which
Dovepress

has also been accepted for indexing on PubMed Central. The manuscript management system is completely online and includes a very quick and fair peer-review system, which is all easy to use. Visit http://www.dovepress.com/testimonials.php to read real quotes from published authors. 